\title{
Hemispheric specialization for communicative processing: neuroimaging data on the role of the right hemisphere
}

\author{
Rochele Paz Fonseca ${ }^{1}$, Lilian Cristine Scherer $^{2}$, Camila Rosa de Oliveira ${ }^{3}$ and Maria Alice de \\ Mattos Pimenta Parente ${ }^{3}$ \\ 1 Pontifícia Universidade Católica do Rio Grande do Sul, Brazil. \\ 2 Universidade de Santa Cruz do Sul, Brazil. \\ 3 Universidade Federal do Rio Grande do Sul, Brazil
}

\begin{abstract}
Neuropsychology has traditionally studied language emphasizing the exclusive control of the left hemisphere of the brain over this process. With the growing development of this area in psychology and the availability of neuroimaging techniques, a critical analysis of the traditional concept of cerebral dominance for language and of the bases of the neurobiological representations of this cognitive function is crucial. In this context, this review aims to investigate evidence brought by neuroimaging studies on the role of the right hemisphere in communicative processing in healthy individuals. Data suggest a co-activation of brain hemispheres in this processing, with an important contribution given by the right hemisphere in discursive, pragmatic-inferential, and prosodic processing.Keywords: communication, pragmatics, discourse, prosody, right hemisphere, cerebral dominance.
\end{abstract}

Received 14 April 2009; received in revised form 6 June 2009; accepted 6 June 2009. Available online 29 June 2009

\section{Introduction}

The interest in the neurobiological bases of linguistic processing dates back to over a century, more specifically, to 148 years ago. In 1861, Paul Broca, in a presentation of two patients in Société d'Anatomie (Dronkers, Plaisant, Iba-Zizen, \& Cabanis, 2007), neuropsychology emerges, together with the concept of cerebral dominance. According to Castro-Caldas (1999), this concept refers to the notion that language is localized in one of the hemispheres, considered to be the dominant - the left hemisphere ( $\mathrm{LH})$, while the right hemisphere $(\mathrm{RH})$ would have less importance. In a general perspective, LH dominance for language is still nowadays confirmed in neuropsychological studies,

RochelePazFonseca,ClinicalandExperimentalNeuropsychology Group, Institute of Psychology, Pontifícia Universidade Católica do Rio Grande do Sul, Brazil. Lilian Cristine Scherer, Department of Linguistics and Literature, Universidade de Santa Cruz do Sul, Brazil. Camila Rosa de Oliveira and Maria Alice de Mattos Pimenta Parente, Institute of Psychology, Universidade Federal do Rio Grande do Sul, Brazil. Correspondence regarding this article should be directed to: Rochele Paz Fonseca, Pontificia Universidade Católica do Rio Grande do Sul, Avenida Ipiranga, 6681, prédio 11, sala 938. CEP: 90619-900, Porto Alegre/RS. Brazil. Phone / Fax: +55 51 3320- 3500 ext. 7745. E-mail: rochele.fonseca@pucrs.br recorded together with some level of $\mathrm{RH}$ activation. The majority of the world population presents LH dominance and, according to Blake (2007), the extent of $\mathrm{RH}$ contribution in linguistic processing has not been precisely and sufficiently investigated to date.

Our knowledge about hemispheric specialization has developed from three complementary sources of investigation: 1) studies with calosotomized or hemispherictomizedindividuals, 2) braindamage studies, and 3) neuroimaging studies with neurologically healthy populations. Studies from the first source have been developed based on the performance of epileptic individuals, to whom the treatment chosen was the section of the corpus callosum (callosotomy), the largest cerebral structure for inter-hemispheric association or, in more acute cases, the total extraction of one of the hemispheres (hemispherectomy). The investigations of the second source have focused on functional sequeli caused by lesions (for example, a stroke or a traumatic brain injury) whose location allows for the inference that the functions partially or totally impaired were executed by the lesioned area. The third source of studies generally investigates the areas with higher blood recruitment, that is, with more substantial activation in the execution of an experimental task. In this way, while the two former sources allow for the generation of inferences 
based on a natural neurological impairment which isolates certain encephalic areas, the latter permits the investigation of functions in an intact brain by means of neuroimaging evidence.

Since the 1990s, a period known as the brain decade, neuroimaging techniques have undergone an impressive advance (Gernsbacher \& Kaschak, 2003). Several studies have applied them in the investigation of linguistic processing in callosotomized, hemispherectomized and brain-lesioned individuals, as well as in neurologically healthy individuals. This study is aimed at reviewing evidence of the role of the RH in communicative processing as revealed by neuroimaging studies with neurologically normal participants. Besides, it aims to critically discuss and rethink the traditional notion of cerebral dominance for language and of the neurobiological bases of human communication, proposing an initial schematic representation of RH areas important for some types of communicative processing, specifically the pragmatic, lexical-semantic, prosodic, and discursive ones.

In order to promote this critical-reflexive analysis, this review article emphasizes two theoretical aspects: 1) cerebral dominance and hemispheric specialization; and 2) functional neuroimaging studies on communicative processing in neurologically healthy adults.

\section{Cerebral dominance and hemispheric specialization}

The origin of the notion of cerebral dominance is interconnected with the birth and development of neuropsychology. Broca's anatomo-clinical study, which pointed out the LH as being dominant for language, is considered the initial landmark of neuropsychology (Springer \& Deutsch, 1997). Aphasiology - the study of the relationships between brain and language, mainly of acquired deficits in this function - aphasia - made possible the construction of the concept of a language zone. This zone was conceptualized by Dejerine in 1914 (Ardila, 1999), corresponding to the perisylvian area. According to this initial notion, Broca's, Wernicke's and the arcuate fasciculus areas were included (Lebrun, 1983). Later on, non-specific associative cortical areas localized in the vicinity of these classical zones, started to integrate the cerebral representation of the linguistic function. Moreover, starting from the second half of the 20th century, relationships between LH subcortical regions and RH cortical regions and language were investigated as well (Démonet, Thierry, \& Cardebat, 2005; Stowe, Haverkof, \& Zwarts, 2005).

The concept of cerebral dominance emerged with the establishment, in the 19th century, of the notion that there was a hemisphere - more important or dominant - responsible for language, while the other hemisphere - less important or non-dominant - did not contribute to this function. The case studies presented by Broca and Wernicke historically marked LH dominance for language. This initial reductionist view postulating the inexistence of a RH contribution to the linguistic function was substituted by a less extremist localizationist view, which postulated that the dominant hemisphere contained the most important language structures, while the counter-lateral hemisphere also contained some structures related to this function, though less important ones. Nowadays, due to the influence of a functionalist or systemic globalist trend, the predominant idea is that language is a dynamic process derived from integrated functions of the whole brain.

This notion of interhemispheric cooperation, still under development, has emerged due to some historical changes. Since the first report of a link between RH damage and communication impairment was written by Eisenson (1959), there was a relevant increase in the number of pragmatic psycholinguistic models (for examples, please see Austin, 1962; Searle, 1969; Bates, 1976; Fauconnier, 1994; Fauconnier \& Turner, 2003), as well as of neuroimaging techniques since the second half of 20th century (for a review, please see Savoy, 2001). This way, after the Second World War, the impaired cognition and communication of several brain damaged individuals could be explored in depth. All this theoretical and methodological evolution contributed to the concept that both hemispheres are important for language production and comprehension (Côté, Payer, Giroux, \& Joanette, 2007).

Thus, since the 1950s, besides the correlates between clinical manifestations of aphasia and lesions in regions of the brain hemisphere dominant for language ( $\mathrm{LH}$, in general), clinical manifestations of communicative difficulties following impairment to the right cerebral region have been mentioned in the literature (for an example, please see Joanette, Goulet, \& Hannequin, 1990). For the past six decades approximately, specific symptoms of impaired communicative abilities have been reported as integrating the RH syndrome, defined as a set of cognitive and communicative impairments originating from RH brain damage (Brookshire, 2003).

Considering the hemispheric specialization for different cognitive functions, there is a certain consensus in the literature nowadays. The LH is considered to be responsible for linguistic thought, analytic reasoning, as well as for verbal memory and language comprehension. Whereas the $\mathrm{RH}$ is related to the following cognitive functions: attention and visuo-spatial perception, body schema, social and emotional intelligence, recognition of facial expressions, among others (Myers, 2001).

The hemispheric specialization referring to language is related to the identification of its components (Bogen, 1997). Table 1 presents a brief definition of each language component, according to Harley (2001), its classification as structural linguistic aspect (related to the language form and grammatical structure) or functional aspect (related to language use and to interlocutors' intentions) and their hemispheric relationships.

Despite some divergences in the literature, most authors, through meta-analyses or empirical studies, identify difficulties in lexical-semantic, discursive, 
Table 1. Language components and their hemispheric representations.

\begin{tabular}{|c|c|c|c|c|c|}
\hline Components & Definitions & $\begin{array}{l}\text { Structural } \\
\text { aspect }\end{array}$ & $\begin{array}{l}\text { Functional } \\
\text { aspect }\end{array}$ & $\mathbf{L H}$ & RH \\
\hline Phonological & Related to the language sounds & $\mathrm{X}$ & & $\mathrm{X}$ & \\
\hline Morphologic & $\begin{array}{l}\text { Related to morphemes - smaller meaningful units } \\
\text { - which build up words }\end{array}$ & $\mathrm{X}$ & & $X$ & \\
\hline Syntactic & Related to sentence organization & $\mathrm{X}$ & & $X$ & \\
\hline Semantic & $\begin{array}{l}\text { Related to the meaning of linguistic units: literal } \\
(\mathrm{LH}) \text { and nonliteral }(\mathrm{RH})\end{array}$ & $\mathrm{X}$ & $\mathrm{X}$ & $\mathrm{X}$ & $\mathrm{X}$ \\
\hline Pragmatic & $\begin{array}{l}\text { Related to the interlocutors' communicative } \\
\text { intentions }\end{array}$ & & $\mathrm{X}$ & & $\mathrm{X}$ \\
\hline
\end{tabular}

pragmatic-inferential and/or prosodic processing in $\mathrm{RH}$ brain-damaged individuals (Côté et al., 2007). However, neuroimaging studies with neurologically impaired populations, heterogeneous samples and varying experimental paradigms contribute to some contrasting results concerning the cerebral areas recruited for the language tasks proposed. Thus, improvements in neuroimaging studies with non-brain-damaged individuals may be useful to a better understanding of the role of the $\mathrm{RH}$ in communication processing.

\section{Neuroimaging studies on communicative processing}

Functional neuroimaging studies mentioned in this review have been predominantly developed using Electroencephalography (EEG), Positron Emission Tomography (PET), or functional Magnetic Resonance Imaging (fMRI). EEG records electrical activity along the scalp produced by the activation of neurons in the brain. The multiple electrodes placed on the scalp record the brain's electrical activity over a short period of time. A derivative of the EEG technique is known as event-related potentials (ERP), which represent the averaged EEG responses time-locked during complex stimuli processing, such as language processing.

PET and fMRI neuroimaging techniques are based on the notion that an increase in the neural activity in a certain cerebral region is accompanied by an increase in blood recruitment in this area. The method mostly adopted for the establishment of inferences on the relationship between changes in the neural activity and the execution of a determined task is known as subtraction. It is the calculation of the subtraction of the blood volume index in the activated area from the blood volume index recorded during the execution of a control task in the same area activated during the execution of the target task (Gernsbacher \& Kaschak, 2003). If the aim is, for instance, to examine the neurobiological substrates for semantic processing of abstract and concrete words, a control task to reduce the impact of the motor artifact generated by the vocalization of the words would be the verbalization of sounds similar to those of speech (such as bababa); in this way, by subtracting the blood volume measured during the control task from the volume measured during the processing of the target task, it would be possible to infer that the areas resulting from this subtraction would correspond specifically to the ones responsible for semantic processing of abstract or concrete words. The careful establishment of a reliable baseline is determinant for an effective subtraction method. However, it is important to state that the subtraction method has several limitations, as discussed by Townsend e Ashby (1983).

In fMRI, hemispheric dominance has generally been indicated by a measure called laterality index. Some methodological issues should be taken into account when calculating this index, namely the nature of the quantification of the left and right hemispheres, the localization of volumes of interest in each hemisphere, the choice of experimental and baseline conditions, the reproducibility of lateralization index values, the dependence on statistical thresholds, and others (Seghier, 2008).

The majority of the neuroimaging experiments have presented a localizationist perspective due to their attempt to pinpoint the specific areas activated for each linguistic component, which occurs within a stronger or weaker relation to the context of language use. Therefore, the investigation to be developed may have a higher or lower structural perspective, that is, more strongly or weakly based on tasks which investigate linguistic components as manifested in socio-interactive situations, where the interlocutors assume their roles as producers of meaning. As an illustration, some studies developed in a structural perspective of language are presented. Binder (1997), by studying processing at the phonemic level, identified bilateral activation in superior and middle regions of 
the temporal lobe. In a study of language processing at the word level, Chee, Tan and Thiel (1999) observed activation in the frontal inferior and supplemental motor area in the LH, as well as in the right cerebellum, during the execution of a word completion task.

Studies such as those presented above tend to generate a predominantly LH activation, since this hemisphere is dominant in processing the structural aspects of language. However, in the study of the different levels of linguistic processing (phonemic, word, sentence, and discourse), the higher the degree of demand for lexical-semantic, discursive, pragmaticinferential and prosodic abilities, the greater the contribution of the RH can be. In other words, the level of $\mathrm{RH}$ activation depends on the nature of the targettask. For instance, if the research aim is to investigate literality of sentences, a higher activation will probably be observed in RH areas while metaphoric sentences are presented. Thus, for a better understanding of the specific role of the RH in communication processing, a review of studies which investigate specifically each of the referred communicative components in healthy subjects, with intact brain abilities, seems to be crucial.

\section{Lexical-semantic processing}

Regarding lexical-semantic processing, that is, language comprehension and production processed predominantly at the word level of semantics, two abilities will be discussed: lexical recall or verbal fluency and semantic judgment. Lexical recall corresponds to the oral or written production of the highest possible quantity of words in a determined time interval. Semantic judgment is represented by the ability of judging the semantic relationship existing or not between two or more words or linguistic expressions (Joanette et al., 1990).

Wood, Saling, Abbott and Jackson (2001) investigated the participation of both hemispheres in lexical orthographic retrieval (word production from a given grapheme). Their fMRI study revealed a bilateral activation of the frontal lobes in this task, with a predominance of LH. The involvement of both cerebral hemispheres has as well been verified by Brickman et al. (2005) in their EEG study. In the processing of lexical orthographic retrieval and lexical semantic retrieval tasks (retrieval of words belonging to the same semantic field, for instance, animals), activation of bilateral frontal and temporal regions was recorded. Blacker, Byrnes, Mastaglia and Thickbroom (2006), however, in a semantic verbal fluency task with adjectives within the animal category, have found frontal activation in both hemispheres, including Broca's area in the LH and a homologous area in the RH. Thus, it seems that the well established semantic-temporal and orthographic-frontal relation is being reviewed, regarding both inter- and intra-hemispheric paradigms.
Concerning the ability to perform semantic judgment, the studies demonstrated a more specific participation of RH. Chan et al. (2004) questioned the predominant participation of LH inferior pre-frontal and medial-superior temporal regions in lexical-semantic processing. By criticizing the exclusive selection of stimuli with precise and dominant meanings in neuroimaging studies with brain-damaged populations, they controlled this variable in their research, comparing neural activation for meanings modulated by lexical ambiguity. In this way, the researchers found activation in LH frontal dorso-lateral regions and in the RH anterior part of the cingulate gyrus and inferior parietal regions when the participants were asked to silently generate a word semantically related to a word with an ambiguous meaning. Conversely, when the word read presented a precise, unique meaning, only LH inferior pre-frontal and medial-superior temporal regions were recruited.

Such findings were corroborated by Titone and Salisbury (2004), who investigated cerebral activation by means of event-related potentials (ERPs) in lexical decision processing between two words more or less related to a third target word, being the conceptual cues manipulated. The stronger the meaning dependence between the words, the higher the activation captured by electrodes located on $\mathrm{RH}$, which demonstrates the role of this hemisphere in the establishment of semantic relationships between two or more words linked by a global context (for example, the relation between finances, banks and money). Oullet-Plamondon, Monchi, Senhadji and Joanette (2005), consonant with this study, observed an increase in bilateral activity in the pre-frontal cortex during the judgment of less prototypical words belonging to the semantic class of animals, that is, more complex words in terms of semantic processing (for example, herring - lower prototypicality in the semantic field of animals - and monkey - higher prototypicality). Activation in the right frontal cortex (inferior frontal gyrus) was also registered in a similar task of semantic judgment in an fMRI study performed by Walter, Jbabdi, Marrelec, Benali and Joanette (2005). In this study, the left medial and inferior frontal gyri and the cuneus were also recruited.

The study developed by Ilg et al. (2007) investigated semantic judgment by proposing the following task: three words should be classified as coherent or incoherent. After the judgment, the participant should name a word with a semantic relationship with all the three words presented. An fMRI analysis identified activations in ventrolateral and frontomedial pre-frontal cortex regions predominantly in the LH. Activation was as well observed in the right dorsolateral pre-frontal cortex; left parietal, temporal and occipital cortices, bilateral activation of the medial occipital cortex; left lingual gyrus; thalamus and cerebellum.

During lexical retrieval and semantic judgment tasks, a bilateral activation predominantly in the LH has been observed. However, although an important participation 
of the $\mathrm{LH}$ is expected in language processing at the word level, the variable complexity of the stimuli seems to be essential to determine the recruitment of $\mathrm{RH}$ regions in semantic judgment tasks. Thus, the higher the stimuli complexity is, represented by non-literality, abstraction, prototypicality, lexical ambiguity, and dependence to a global context, the higher tends to be RH recruitment.

\section{Discourse processing}

Discourse is conceptualized by Chapman, Highley and Thompson (1998) as being a language unit formed by more than one sentence, used to transmit a message, demanding linguistic, pragmatic, and cognitive functions. At the linguistic level, it requires grammatical processing for language comprehension or production, at its more superficial, structural level; at the pragmatic level, it requires the processing of the interlocutors' intentions and of more complex inferences (analyzed in the next subsection); and, at the cognitive level, it demands the processing by other cognitive functions, such as memory, attention, and problem solution. In this review, only one type of discourse will be analyzed narrative discourse - since this is the type mostly studied by neuropsycholinguists. Narrative discourse consists of the description of a series of actions and events causally and chronologically connected. It is important to emphasize that for an adequate comprehension and production of this and other types of discourse the various linguistic components (phonological, morphological, syntactic, semantic, and pragmaticdiscursive) must be processed by the interlocutors.

A growing number of neuroimaging studies on discourse has aimed to investigate the processing of implicit language for effective discourse production and comprehension. In an fMRI experiment, Vogeley et al. (2001) studied the theory of mind, that is, the ability of comprehending the interlocutor or the characters' mental states in the narrative discourse processing. They compared cerebral activity generated during the reading of stories with and without implicit messages to the reading of sentences not interrelated. When there was an implicit message to be understood, $\mathrm{RH}$ activation was observed (anterior cingulate giry, temporo-parietal region, pre-motor and motor cortex). Xu, Kemeny, Park, Frattali and Braun (2005) designed an experiment manipulating the context variable in three experimental conditions with a growing demand level on non-literal processing: non-connected sentences, connected sentences, and brief narratives. The RH was gradually activated in accordance with the increase of contextual complexity, with the following areas activated at the narrative level: bilateral extrasylvian areas - precuneus, medial pre-frontal region and temporo-parieto-occipital cortex.

According to these and similar studies, there is evidence for the occurrence of a more intense and/ or diffuse activation of the $\mathrm{RH}$ in narrative discourse processing, with the growth of complexity or demand of non-literal language processing. In other words, the level of activation and the amplitude of coverage of $\mathrm{RH}$ areas vary as a function of the manipulation, for instance, of the complexity of the inferences required.

\section{Pragmatic-inferential processing}

Some of the communicative functions linked to pragmatic abilities are inferential or pragmaticinferential processing. Inferences consist of mental representations constructed by the reader or listener during discourse comprehension, by the application of his/her own knowledge to the explicit evidence provided by the message (Gutiérrez-Calvo, 1999; Dascal, 2006). The explicit textual information, connected to previous knowledge relevant to the understanding of the linguistic content, allows the reader/listener to infer, that is, to understand implicit information. All kinds of tasks which involve non-literal content, such as humor, metaphors, and indirect speech acts, require the processing of contextual inferences (Joanette et al., 1990; Beeman, 1993). In this review, as an illustration, only the pragmatic-inferential ability of metaphor comprehension will be discussed. Metaphor is defined as being the figure of speech expressing a connotative meaning, describing a knowledge domain by using the concept of another domain which originally has a different literal meaning (Harley, 2001).

Among neuroimaging studies on pragmaticinferential processing, Bottini et al. (1994) investigated the role of the $\mathrm{RH}$ in the interpretation of new metaphors, that is, metaphors with low familiarity, with no automatic comprehension, in normal individuals, by using PET. The participants solved three tasks: metaphoric sentences and literal sentences analysis and lexical decision. They had to judge sentences' plausibility. LH areas were activated during the analysis of both literal and metaphoric sentences. However, in metaphor comprehension, a large number of RH areas were activated: pre-frontal cortex, medial temporal gyrus, precuneus, and the posterior part of the cingulate gyrus. The study developed by Mashal, Faust and Hendler (2005) corroborated this result, but with a specificity not mentioned yet: $\mathrm{RH}$ areas were recruited exclusively in the processing of new, less conventional metaphors. Neural networks of activation were recorded with a predominant participation of the right homologous area to Wernicke's.

From a brief analysis of these two studies, as well as of others on inferential stimuli in discourse, there is evidence to suggest a bilateral activation in pragmatic-inferential processing. It seems that the lower conventionality and familiarity of metaphorical sentences are, the more evident is the amount and specificity of RH activation. This specificity was 
referred to in studies with linguistic stimuli elaborated with high psycholinguistic rigor, such as the study developed by Mashal et al. (2005).

\section{Prosodic processing}

Prosody is an important communicative element, pointed as one of the main extralinguistic attributes present in oral communication. It consists of the melodic line of discourse, produced by the variation in frequency, rhythm, and emission emphasis (Harley, 2001). In this review, besides studies with general prosodic characteristics, a specific type will be approached - emotional prosody, which corresponds to a set of intonations which differentiate the feelings expressed in linguistic units, such as sadness, happiness, anger, surprise, etc. (Joanette at al., 1990; Mitchell, 2007).

In an fMRI study on prosodic processing with normal individuals, Hesling, Clément, Bordessoules and Allard (2005) aimed to investigate the brain mechanisms involved in the perception of discursive stimuli with high and low levels of expressive prosody. They observed that information transmitted with a high level of prosody expression required a higher specific RH activation in comparison to messages with low level of expressive prosody, with low activation in the LH. A specific participation of the RH was observed in the processing of the fundamental frequency of prosodic modulations.

Besides this study investigating general prosodic characteristics, other studies investigated emotional prosody specifically. In an investigation in which the participant had to judge how positive or negative was a normal discourse (with a linguistic meaning) or a prosodic discourse (without linguistic meaning, only with variations in the prosodic modulations), the following regions were activated in the judgment of the normal discourse: the perisylvian area of the LH and frontal and subcortical areas bilaterally; in the prosodic discourse, though, a higher frontal $\mathrm{RH}$ activation was observed (Kotz et al., 2003). This finding was also registered by Wildgruber et al. (2005) in an fMRI investigation of the identification of emotional intonation (a task of recognition of five different basic emotions in voice - happiness, sadness, anger, fear, and disappointment). Besides bilateral frontal region participation, the RH posterior-superior temporal sulcus was activated. Similarly, Wildgruber, Pihan, Ackermann, Erb and Grodd (2002), in the analysis of the neural activation in the processing of a similar task, observed the recruitment of the mesio-frontal cortex bilaterally and of the RH inferior parietal region.

Although $\mathrm{RH}$ activation in prosodic processing seems to be a consensus in the neuroimaging studies analyzed in this review, some studies point to a coactivation of the left temporal lobe in tasks which evaluate the comprehension of emotional prosody, depending on the complexity of the verbal stimuli (Mitchell \& Ross, 2008). There is a distributed activation in the four RH lobes in tasks that require communicative processing, with a preponderance of activation of the frontal and temporal lobes, mainly of the right temporal region homologous to Wernicke's area, localized in the left temporal lobe. The heterogeneity in the RH activated areas needs to be further addressed, since frontal, temporal, parietal, and occipital regions were recruited in a nonsystematic manner in the investigations mentioned in this review. Moreover, a deep investigation of the role of subcortical RH areas needs to be carried out.

Table 2 presents a synthesis of the results on $\mathrm{RH}$ activation reported in the neuroimaging studies involving the communicative processes reviewed in this article.

In addition, the present paper proposes an initial graphic representation of $\mathrm{RH}$ participation in human communication. In this way, Figure 1 presents two views of a schematic representation of the components of language processing generally recruiting $\mathrm{RH}$ participation and the regions implicated in their processing.

Table 2. Right hemisphere areas activated in the four components of communicative processing.

\section{Processing Right hemisphere activated areas}

Lexical-semantic Inferior frontal gyrus, temporal and inferior parietal lobes, anterior part of the cingulate gyrus, pre-frontal and mesial occipital cortex

Discursive Anterior part of the cingulate gyrus, temporo-parieto-occipital region, medial pre-frontal area, precuneus

Pragmaticinferential

Pre-frontal cortex, medial temporal gyrus, posterior part of the cingulate gyrus, precuneus 

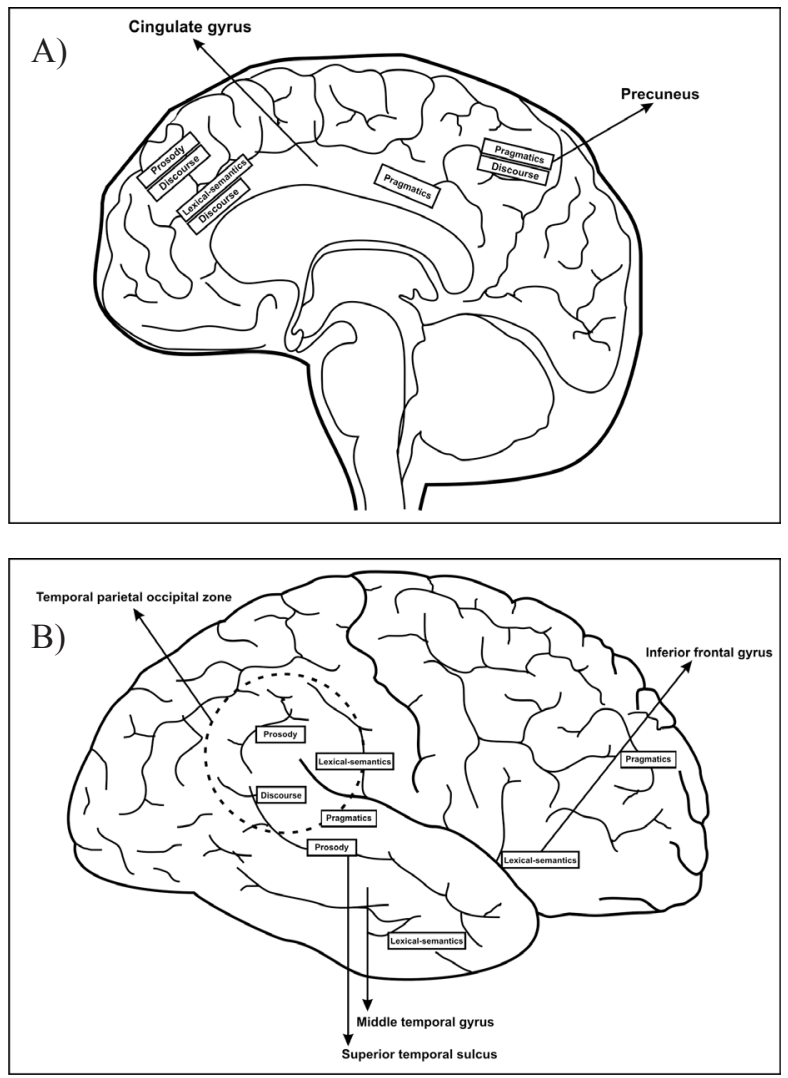

Figure 1. Schematic representation of the components of language processing generally recruiting $\mathrm{RH}$ participation and the regions implicated in their processing (A: sagittal view; B: lateral view).

Finally, it is important to highlight that bilateral activation, with increased participation of certain areas in the RH (generally counter-lateral ones) is also observed in non-linguistic tasks exploring the impact of the level of complexity in the execution of non-verbal tasks (Herd, Banich, \& O'Reilly, 2006; Yoshizaki, Weissman, \& Banich, 2007).

\section{Final comments}

This brief review makes it possible to critically analyze some studies that investigated the hemispheric lateralization in linguistic-communicative functions in non-brain-damaged individuals. The studies reported in the literature have mainly dealt with language in its more structural perspective, while a reduced number has focused on language as a socio-communicative vehicle, with its meanings and discursive-pragmatic aspects. However, it is also necessary to emphasize that the investigation of discursive-pragmatic aspects is still subject to the limitations imposed by neuroimaging techniques, which, in their majority, impose difficulties to the presentation of long stimuli such as texts, or even complex sentences, requiring a very careful planning for presentation and analysis.

The LH dominance for linguistic processing taken as a whole has been confirmed in the literature. However, the concept of hemispheric dominance needs to be redimensioned, since at the phonological, syntactic, and semantic processing, as well as in discursive, pragmaticinferential, and prosodic processing, some degree of neural activation in the RH has been detected. More specifically, the RH seems to play a relevant role in the processing of functional aspects. In other words, empirical evidence suggests that, as far as RH participation on communication tasks is recruited, it is possible to consider an important $\mathrm{RH}$ influence dominance in the processing of functional linguistic components.

Moreover, the findings of neuroimaging studies expand the traditional neuropsychological language model by bringing evidence of activation in cortical areas associated to the LH, RH cortical areas correlated to classical language regions in the LH, and bilateral subcortical areas, as well as cerebellar zones. In this way, the classical language area - left perisylvian region - is not the only one recruited in linguistic-communicative processing.

Regarding the activation of $\mathrm{RH}$ areas in tasks examining the types of communicative processing analyzed above, the following regions have been detected: temporal, parietal, frontal, and occipital cortical regions and subcortical areas - anterior and posterior parts of the cingulate gyrus. The discrepant data on intra-hemispheric location indicate the necessity of further studies on the communicative neurobehavioral relationships in normal individuals. Likewise, there should be a higher concern with stimuli selection, with a control of variables such as task complexity, for instance, which is directly related to other psycholinguistic aspects, such as contextualization level, meaning saliency, abstraction, and others. An attempt to individualize the processing steps is essential, aiming to reach greater proximity with theoretical psycholinguistic models. This may allow researchers to identify a temporal hierarchy in the activation of $\mathrm{RH}$ regions that could at least partially justify the heterogeneous findings.

Important data on neurofunctional models regarding temporal hierarchy, such as those investigating functional connectivity and tractography, have been developed (Glasser \& Rilling, 2008). Equally, techniques such as fNIRS (functional Near-Infrared Spectroscopy) and ERP (Event Related Potentials) certainly represent relevant tools in the investigation of temporal issues regarding the functioning of the brain circuitry, since their temporal definition is in the order of milliseconds, and thus higher than fMRI and PET. As an illustration, studies developed by Friedericci (2002), Palolahti, Leino, Jokela, Kopra and Paavilainen (2005), and Friedericci and Weissenborn (2007) were able to distinguish temporal activation of semantic and syntactic processing, which allowed these researchers to speculate on neurolinguistic theories concerning the dissociation or not of these two types of linguistic processing. Therefore, further studies should concomitantly investigate temporal and special neuronal processing 
by applying different and complementary neuroimaging techniques, so as to obtain a deeper understanding of cognitive processing in the brain, including language. Moreover, these investigations should also encompass language processing in its communicative facet, going beyond the structural aspects of language, to focus on language from a pragmatic and discursive perspective.

By means of the increasing repertory of neuroimaging resources and their refinement, together with a growing methodological rigor in experimental and baseline tasks, more solid knowledge on the neurobiological bases of communicative processing will certainly be reached. Finally, awareness should be demonstrated in order to avoid the tendency to defend the opposite extreme of the traditional concept of cerebral dominance, that is, one cannot postulate the exclusivity of the $\mathrm{RH}$ in processing lexicalsemantic, discursive, pragmatic-inferential, and prosodic components. As suggested in the literature, an inter- and intra-hemispheric cooperation seems to be crucial in such processes. A linguistic dominance for the LH regarding structural aspects (phonological, syntactic, and literal semantic) could be proposed, as well as a cerebral dominance for the RH when it comes to functional or context aspects (pragmatic, prosodic, and nonliteral semantics), towards an interand intra-hemispheric cooperation.

\section{References}

Ardila, A. (1999). The role of insula in language: an unsettled question. Aphasiology, 13, 79-87.

Austin, J.L. (1962). How to do things with words. Oxford: Oxford University Press.

Bates, E. (1976). Language and context: The acquisition of pragmatics. New York: Academic Press.

Beeman, M. (1993). Semantic processing in the right hemisphere may contribute to drawing inferences from discourse. Brain and Language, 44, 80-120.

Binder, J.R. (1997). Neuroanatomy of language processing studied with functional MRI. Clinical Neuroscience, 4, 87-94.

Blake, M.L. (2007). Perspectives on treatment for communication deficits associated with right hemisphere brain damage. American Journal of Speech - Language Pathology, 16, 331-342.

Blaker, D., Byrnes, M.L., Mastaglia, F.L., \& Thickbroom, G.W. (2006). Differential activation of frontal lobe areas by lexical and semantic language tasks: A functional magnetic resonance imaging study. Journal of Clinical Neuroscience, 13, 91-95.

Bogen, J.E. (1997). Does cognition in the disconnected right hemisphere require right hemisphere possession of language? Brain and Language, 57, 12-21.

Bottini, G., Corcoran, R., Sterzi, R., Paulesu, E., Schenome, P., Scarpa, P., Frackowiak, R.S., \& Frith, C.D. (1994). The role of the right hemisphere in the interpretation of figurative aspects of language: A positron emission tomography activation study. Brain, 117, 1241-1253.

Brickman, A.M., Paul, R.H., Cohen, R.A., Williams, L.M., Macgregor, K.L., Jefferson, A.L., Tate, D.F., Gunstad, J., \& Gordon, E. (2005). Category and letter verbal fluency across the adult lifespan: Relationship to EEG theta power. Archives of Clinical Neuropsychology, 20, 561-573.

Brookshire, R.H. (2003). Introduction to neurogenic communication disorders. Missouri: Mosby.

Castro-Caldas, A. (1999). A herança de Franz Joseph Gall. Lisboa: McGraw-Hill.
Chan, A.H.D., Liu, H., Yip, V., Fox, P.T., Gao, J., \& Tan, L.H. (2004). Neural systems for word meaning modulated by semantic ambiguity. Neuroimage, 22, 1128-1133.

Chapman, S.B., Highley, A.P., \& Thompson, J.L. (1998). Discourse in fluent aphasia and Alzheimer's disease: Linguistic and pragmatic considerations. Journal of Neurolinguistics, 11(1-2), 55-78.

Chee, J.D., Tan, E.W., \& Thiel, T. (1999). Mandarin and English single word processing studied with functional magnetic resonance imaging. Journal of Neuroscience, 19, 3050-3056.

Côté, H., Payer, M., Giroux, F., \& Joanette, Y. (2007). Towards a description of clinical communication impairment profiles following right-hemisphere damage. Aphasiology, 21(6, 7,8), 739-749.

Dascal, M. (2006). Interpretação e compreensão. São Leopoldo: UNISINOS.

Démonet, J.F., Thierry, G., \& Cardebat, D. (2005). Renewal of the neurophysiology of language: functional neuroimaging. Psychological Reviews, 85, 49-95.

Dronkers, N.F., Plaisant, O., Iba-Zizen, M.T, \& Cabanis, E.A. (2007). Paul Broca's historic cases: high resolution MR imaging of the brains of Leborgne and Lelong. Brain, 130, 1432-1441.

Eisenson, J. (1959). Language dysfunctions associated with right brain damage. American Speech and Hearing Association, 1: 107.

Fauconier, G. (1994). Mental spaces: Aspects of meaning construction in natural language. Cambridge: MIT Press.

Fauconnier, G., \& Turner, M. (2003). The way we think: Conceptual blending and the mind's hidden complexities. New York: Basic Books.

Friederici, A.D. (2002). Towards a neural basis of auditory sentence processing. Trends in Cognitive Sciences, 6, 78-84.

Friederici, A.D., \& Weissenborn, J. (2007). Mapping sentence form onto meaning: the syntax-semantic interface. Brain Research, $1146,50-58$.

Gernsbacher, M.A., \& Kaschak, M.P. (2003). Neuroimaging studies of language production and comprehension. Annual Review of Psychology, 54, 91-114.

Glasser, M.F., \& Rilling, J.K. (2008). DTI tractography of the human brain's language pathways. Cerebral Cortex, 18, 2471-2482.

Gutiérrez-Calvo, M. (1999). Inferencias en la comprensión del lenguaje. In M. Veja \& F. Cuetos (Eds.), Psicolinguistica del español (pp. 231-270). Madrid: Trotta.

Harley, T. (2001). The psychology of language. New York: Psychology Press.

Herd, S.A, Banich, M.T., \& O'Reilly, R.C. (2006). Neural mechanisms of cognitive control: An integrative model of Stroop task performance and fMRI data. Journal of Cognitive Neuroscience, 18, 22-32.

Hesling, I., Clement, S., Bordessoules, M., \& Allard, M. (2005). Cerebral mechanisms of prosodic integration: Evidence from connected speech. Neuroimage, 24, 937-947.

Ilg, R., Vogeley, K., Goschke, T., Bolte, A., Shah, J.N., Pöppel, E., \& Fink, G.R. (2007). Neural processes underlying intuitive coherence judgments as revealed by fMRI on a semantic judgment task. Neuroimage, 38, 228-238.

Joanette, Y., Goulet, P., \& Hannequin, D. (1990). Right hemisphere and verbal communication. New York: Springer.

Kotz, S.A., Meyer, M., Alter, K., Besson, M., Von Cramon, D.Y., \& Friederici, A.D. (2003). On the lateralization of emotional prosody: An event-related functional MR investigation. Brain and Language, 86, 366-376.

Lebrun, Y. (1983). Tratado de afasia. São Paulo: Panamed.

Mashal, N., Faust, M., \& Hendler, T. (2005). The role of the right hemisphere in processing nonsalient metaphorical meanings: Application of principal components analysis to fMRI data. Neuropsychologia, 43, 2084-2100.

Mitchell, R.L. (2007). fMRI delineation of working memory for emotional prosody in the brain: Commonalities with the lexicosemantic emotion network. Neuroimage, 36, 1015-1025.

Mitchell, R.L., \& Ross, E.D. (2008). fMRI evidence for the effect of verbal complexity on lateralisation of the neural response associated with decoding prosodic emotion. Neuropsychologia, 46, 2880-2887.

Myers, P.S. (2001). Toward definition of RHD syndrome. Aphasiology, 15(10/11), 913-918.

Oullet-Plamondon, C., Monchi, O., Senhadji, N., \& Joanette, Y. (2005). Traitement sémantique: La complexité accroît l'activation cérébrale chez les jeunes adultes [Abstracts]. In Sociedade Latinoamericana de Neuropsicologia (Ed.) Abstract of the IX SLAN meeting. Cartagena, Colômbia: SLAN. 
Palolahti, M., Leino, S., Jokela, M., Kopra, K., \& Paavilainen, P. (2005). Event-related potentials suggest early interaction between syntax and semantics during on-line sentence comprehension. Neuroscience Letters, 384, 222-227.

Savoy, R.L. (2001). History and future directions of human brain mapping and functional neuroimaging. Acta Psychologica, 107(1-3), 9-42.

Searle, J.R. (1969). Speech Acts. Cambridge: Cambridge University Press. Seghier, M.L. (2008). Laterality index in functional MRI: Methodological issues. Magnetic Resonance Imaging, 26, 594-601.

Springer, S.P., \& Deutsch, G. (1997). Left brain, right brain. New York: W.H. Freeman.

Stowe, L.A., Haverkof, M., \& Zwarts, F. (2005). Rethinking the neurological basis of language. Lingua, 115, 997-1042.

Titone, D.A., \& Salisbury, D.F. (2004). Contextual modulation of N400 amplitude to lexically ambiguous words. Brain and Cognition, 55, 470-478.

Townsend, J.T., \& Ashby, F.G. (1983). The Stochastic modeling of elementary psychological processes. Cambridge, Cambridge University Press.

Vogeley, K., Bussfeld, P., Newen, A., Hermann, S., Happé, F., \&
Falkai, P. (2001). Mind reading: Neural mechanisms of Theory of Mind and self-perspective. Neuroimage, 14, 170-181.

Walter, N., Jbabdi, S., Marrelec, G., Benalti, H., \& Joanette, Y. (2005). fMRI brain interactivity analysis of phonological and semantic word processing. In Human Brain Mapping (Ed.) Abstract of the HBM. Toronto, Canadá: HBM.

Wildgruber, D., Pihan, H., Ackermann, H., Erb, M., \& Grodd, W. (2002). Dynamic brain activation during processing of emotional intonation: Influence of acoustic parameters, emotional valence, and sex. Neuroimage, 15, 856-869.

Wildgruber, D., Riecker, A., Hertrich, I., Erb, M., Grodd, W, Ethofer, T., \& Ackermann, H. (2005). Identification of emotional intonation evaluated by fMRI. Neuroimage, 24, 1233-1241.

Wood, A.G., Saling, M.M., Abbott, D.F., \& Jackson, G.D. (2001). A neurocognitive account of frontal lobe involvement in orthographic lexical retrieval: An fMRI study. Neuroimage, 14, 162-169.

Xu, J., Kemeny, S., Park, G., Frattali, C., \& Braun, A. (2005). Language in context: Emergent features of word, sentence, and narrative comprehension. Neuroimage, 25, 1002-1015.

Yoshizaki, K., Weissman, D.H., \& Banich, M.T. (2007). A hemispheric division of labor aids mental rotation. Neuropsychology, 21, 326-36. 